DE83 009136

\title{
DISCLAIMER
}

This report was prepared as an account of work sponsored by an agency of the United States Government. Neither the United States Government nor any agency thereof, nor any of their employees, makes any warranty, express or implied, or assumes any legal liability or responsibility for the acruracy, completeness, or usefulness of any information, apparatus, product, or process disclosed, or represents that its use would not infringe privately owned rights. Reference herein to any specific commercial product, process, or service by trade name, trademark, manufacturer, or otherwise does not necessarily constitute or imply its endorsement, recommendation, or favoring by the United States Government or any agency thereof. The views and opinions of authors expressed herein do not necessarily state or reflect those of the United States Government or any agency thereof. 


\section{Ultra-relativistic Heavy Ions and the CBA}

The study of ultra-relativistic heavy ions at an accelerator such as the CBA provides a unique glimpse of matter as it may have appeared in the early universe. This hot dense matter very probably appears as a quark-gluon plasma which expands and cools into hadronic matter. The theoretical issues which may be tested by studying this matter in a laboratory environment have been extensively discussed in the literature, and I shall not discuss them here. 1

The CBA would provide data at the very highest energies, and produce matter at the highest energy densities. The possibility of using a cyclotron to inject very heavy ions into the AGS and then into the $\mathrm{CBA}^{2}$ would also allow the production of quark-gluon matter at higher energy densities than would light ions, and would make the matter in a larger volume where surface effects are minimized. At the highest energies with very heavy ions, there is great flexibility in the experimental signals which might be studied, as well as the nature of the matter which is produced. ${ }^{1,3-5}$ The CBA provides a wide enough kinematic range so that the central region may be studied. The fragmentation region and central region provide different environments where a plasma might form. The former is baryon rich and might be studied at SPS energies as well as at the CBA. The central region is at high temperature with smal.1 baryon density and is not accessible for very heavy ions at the SPS. Obtaining a clearly defined central region provides a probe of new physics, and center-of-mass energies as low as $50 \mathrm{GeV} /$ nucleon are adequate for this purpose. Higher energies might also be somewhat useful if there is significant deviation from scaling in the central region. 
Using very heavy ions minimizes contamination of a produced quarkgluon plasma by surface radiation. This contamination is by no means a trivial effect, and its magnitude is in dispute. Ions of very large baryon number A minimize this effect. Also, the energy density produced in the plasma increases with $A$. In the fragmentation region, the increase is expected to be $A^{1 / 3}$, and in the central region may approach a constant for sufficiently large $A$ or may grow as $A^{1 / 3}$ or $A^{2 / 3}$. Since there is some theoretical uncertainty as to the magnitude of energy deposition needed for plasma formation and the magnitude generated by nuclear collisions, using very heavy ions seems to be called for.

The pressing experimental issues for ultra-relativistic nuclear collisions are whether the conditions necessary for the formation of a plasma are produced, and how a plasma might be probed and diagnosed. The meager data available from cosmic ray experiments provide some hint that exciting new phenomena may take place in these collisions, but the results are as yet too scarce to seriously constrain speculation. ${ }^{6}$ In spite of this lack of data, a reasonable theoretical scenario for ultra-relativistic heavy ion collisions has been abstracted from knowledge of high energy hadron-hadron and hadron-nucleus collisions. ${ }^{3-8}$ The production and properties of a quark-gluon plasma have been discussed within this framework, and experimental probes have been presented which provide a qualitative and semi-quantitative analytical framework. All theoretical studies of these collisions suggest that in ultra-relativistic collisions of very heavy ions such as uranium energy densities in excess of 10 times nuclear matter energy density are produced, and there is time for kinetic equilibrium. This energy density is quite probably enough to produce a quark-gluon plasma. Due to the exploratory nature 
of this field, experiments should, of course, be discussed with a flexible and open mind. After all, a new region of physical systems might be studied in a range of energy densities of 10-100 times that about which there is any experimental input. Although dramatic signals for the existence of a quark-gluon plasma might arise, an equally likely possibility is that convincing evidence for a plasma will only appear after a good deal of combined, systematic effort by theorists and experimentalists.

In most treatments of ultra-relativistic nuclear collisions, the interaction of the nuclei in the initial stages of the collision when the quark-gluon plasma might be produced is usually treated as a quasi-incoherent sum of nucleon-nucleon interactions. Some coherence may be allowed for in such treatments by including shielding effects which might modify the dependence of the amount of matter formation on the nuclear baryon number as formation proceeds from the fragmentation region to the central region.

If matter formation proceeds incoherently, a fixed impact parameter might be easily identified. At a fixed impact parameter, a fixed number of nucleons interact. Since fluctuations are small, the total multiplicity as well as the degree of nuclear disintegration are well defined functions of impact parameter.

If impact parameter is well defined, the study of ultra-relativistic nuclear collisions might be facilitated by considering "head-on" collisions, $b \bumpeq$ Ifm, which is $\sim 1 / 2 \%$ of all Uranium-Uranium collisions. Head-on collisions produce the highest multiplicities, and are conceptually simpler than grazing collisions. We shall discuss head-on collisions of very heavy nuclei in the remainder of this report. 
The standard "new scenario" for ultra-relativistic nuclear collisions 3-5,7-8 may be understood by contrast with a model, the "old scenaria", which works at low energies and might apply up to intermediate energies of perhaps 10-15 GeV/N. ${ }^{9-12}$ We shall soon consider a non-standard scenario for which fluctuations are large. ${ }^{13}$ A first function of ultra-relativistic heavy ion experiments is to verify or rule out various models of the collision dynamics. These experiments may illuminate our understanding of the space-time structure of hadronic processes. 14

In the old standard scenario, which is presumably valid at low energies, a nuclear collision is most simply analyzed in the center-of-mass frame. In this frame, the nuclei approach one another with energies $\mathrm{E}_{\mathrm{cm}} / 2$ and are Lorentz contracted to a longitudinal length $R / \gamma$ where $\gamma$ is the Lorentz boost factor to the center-of-mass frame and $R$ is the nuclear radius. This situation is shown in Fig. (1). When these two nuclei strike one another, it is assumed they come to rest in the center-of-mass frame. The longitudinal size is taken to be $2 \mathrm{R} / \mathrm{Y}$, although there are certainly factors of two uncertainty in this longitudinal size scale. This situation is shown in Fig. (2). The energy density achieved is

$$
\begin{aligned}
\varepsilon & =\left(\frac{1}{2 / \gamma}\right)\left(\frac{E_{c m}}{2 A}\right) \frac{2 A}{V} \\
& =\gamma^{2} \rho_{n \cdot m} .
\end{aligned}
$$

where the factors in Eqn. (1) are $V$ as the nuclear volume and $\rho$ as the density of nuclear matter. This configuration of compressed hadronic 
provides initial conditions for hydrodynamic equations which describe the time evolution of the hadronic matter distribution. This model has two distinguishing features which ultra-relativistic collisions could explore. The energy density $\varepsilon$ does not scale with beam energy due to the factor of $\gamma^{2}$ in Eqn. (1), and the rapidity density of baryonic charge, baryons minus anti-baryons, is spread alnost uniformly throughout the kinematically allowed range of rapidity (Fig. (3)).

The old scenario is probably valid up to some energy at which the effects of nuclear transparency become important. This energy has been estimated to be $\mathrm{E}_{\mathrm{cm}} \sim 5 \mathrm{GeV} /$ nucleon by Goldhaber, and energy densities $\varepsilon \sim 10 \varepsilon_{\mathrm{n} . \mathrm{m}}$. times that of nuclear matter, that is $\varepsilon \sim 1-2 \mathrm{GeV} / \mathrm{fm}^{3}$, might be achieved before transparency is significant.

When the nuclei become transparent, the energy density does not suddenly decrease, but simply approaches a limiting constant, and Eqn. (1) is no longer valid. The limiting energy density is presumably close to that given by Eqn. (1) at the maximum energy before transparency occurs, and explicit computations give energy densities $\varepsilon \sim 2 \mathrm{GeV} / \mathrm{im}^{3}$ for the fragmentation region of $\mathrm{U}-\mathrm{U}$ collisions.

The new scenario combines particle production and Lorentz time dilation effects to produce a picture of nucleus-nucleus collisions which incorporates scaling and nuclear transparency. Nuclear transparency is a well established feature of hadron-nucleus interactions. An incident hadron easily punches through a target nucleus and suffers little momentum loss. The distribution of hadrons produced in the projectile fragmentation region is strikingly similar to that of hadron-hadron collisions. The distribution of particles produced in hadron-nucleus collisions, of course, approximately scales. 
In the new scenario, the Lorentz contracted nuclei approach one another with a limiting thickness of $1 \mathrm{fm} .^{14}$ This 1imiting thickness arises because the valence nucleons in the nuclei couple to low longitudinal momentum degrees of freedom, and these degrees of freedom contract to a thickness $\tau \sim 1 / \mathrm{p}^{\prime \prime} \backsim 1 \mathrm{fm}$. The valence nucleons are very energetic and contract to $\tau \sim R / \gamma$ where $\gamma$ is of the order of $\gamma_{\mathrm{cm}}$.

When the two nuclei pass through one another, the low momentum virtual hadronic degrees of freedom of the nuclei materialize. This presumably happens in a natural hadronic time scale, $\Delta \tau \sim 1 \mathrm{fm}$. The valence nucleons and higher momentum virtual hadrons pass through one another since, in their own rest frames, a hadronic time $\Delta \tau \sim$ lfm has not passed. New matter forms as soon as this matter has had a time of $1 \mathrm{fm}$ to materialize in its rest frame. There is therefore a formation zone which is moving towards the valence nucleons (Fig. 4). In the center-of-mass frame the time of formation and its position are correlated with longitudinal momentum since $\tau \sim \mathrm{x} \sim \mathrm{p}^{\prime \prime}$. As time evolves the formation zone approaches the nucleus since the particle is a distance

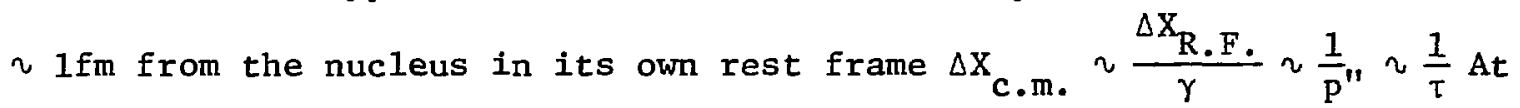
at a time $\tau \sim X \sim E_{\mathrm{cm}}$, the forming matter catches the projectile and target nuclei and the nuclei fragment.

This description of matter formation is called the inside-outside cascade. 14 The name follows from the picture described above where low momentum matter fcrms in the region inside the two nuclei and proceeds outward to catch and fragment them. This cascade structure is a consequence 
of time dilation and the soft nature of hadronization. The inside-outside cascade very generally describes hadronic interactions and has applications for jets in $\mathrm{e}^{+} \mathrm{e}^{-}$collisions, hadron-hadron, and hadron-nucleus collisions. The initial energy density of matter produced in nuclear collisions may be estimated using this simple space-time picture. We know the spacetime points at which matter first materializes in these collisions. If we assume that the matter materializes at is does in a pp collision and take an incoherent sum over A protons distributed in the nuclei, the initial matter distribution is determined. ${ }^{5}$ This assumption might have to be slightly modified in the central rapidity region since shielding or anti-shielding effects might rescale the matter formation by a factor of $A^{-1 / 3}-A^{1 / 3} .^{8}$ Within the uncertainties of this effect, the central region energy density immediately after formation is expected to be $\varepsilon \sim 1-10 \mathrm{GeV} / \mathrm{fm}^{3},{ }^{3-5}$ and $\varepsilon \sim 1-2 \mathrm{GeV} / \mathrm{fm}^{3^{7-8}}$ in the fragmentation region. The energy density increases as $\mathrm{A}^{1 / 3}$ in the fragmentation region so that larger nuelei produce larger energy densities.

The distribution of this matter as it forms provides initial conditions for hydrodynamic equations. ${ }^{5,8,15}$ These equations assume isotropic expansion and only involve the relationship between energy density and pressure since Energy density and pressure may be computed using lattice Monte-Carlo methods, the time evolution of the hadronic matter may be computed with present day technology. The assumption of isotropic expansion is expected to be good as long as the hadronic matter does not expand through a first order phase transition. With first order transition, matter expands approximately isotropically until 
It passes through the transition where large scale density fluctuations and metastable configurations may invalidate hydrodynamics. These phenomena are discussed later, and may provide signals of plasma formation.

Assuming isotropic expansion, Bjorken has shown that $\frac{d N}{d y}$ is invariant in the central region. ${ }^{5}$ The measurement of this quantity allows a determination of the energy density at early stages in the collision when the matter first forms. This follows by assuming $\Delta y \sim 1$ corresponds to $\Delta \mathrm{X}^{\prime \prime} \sim 1 \mathrm{fm}$, as is the case for an inside-outside cascade, and that the typical transverse momentum of particles when they are produced is greater than or of the order of inelastically produced particles in hadron-hadron collisions. As shown by Gyulassy, for a head-on collision $^{16}$

$$
\varepsilon \sim \frac{.1}{A^{2 / 3}} \frac{d N}{d y}
$$

There is data which strongly suggests that energy densities $\varepsilon \gtrsim 3 \mathrm{GeV} / \mathrm{fm}^{3}$ have been acheived in nucleus-nucleus collisions. The JACEE cosmic ray collaboration has observed a 4-5 TeV/nucleon Si-AgBr collision. $^{17}$ The central region multiplicity is $\frac{\mathrm{dN}}{\mathrm{dy}} \sim 200$, and a simple estimate gives $\varepsilon \sim 3 \mathrm{GeV} / \mathrm{fm}^{3}$. (If the transition is first order, this analysis fails, but a quark-gluon plasma would have been produced.) This result strongly suggests that energy densities sufficient for the formation of a plasma exist in ultra-relativistic nuclear collisions. Extrapolation of this energy density to collisions of uranium at CBA give $\varepsilon \sim 6 \mathrm{GeV} / \mathrm{fm}^{3}$, which is 50 times that of nuclear matter. 
A clear signal which distinguishes the new scenario from the old, in addition to scaling, is $\frac{d N}{d y}$ for baryons minus anti-baryons. This distribution has the double humped structure of Fig. 5 in the new scenario, and the width of the nuclear fragmentation region is $\Delta y \sim \ln 2 A^{1 / 3} \sim 3$ units. The pion distribution does not clearly distinguish the old and new pictures, but the $A$ dependence of $\frac{d N}{d y}$ must be known to make reasonable hydrodynamical models and to extrapolate the obtainable energy densities in uranium collisions. Detailed features of specific models of inelastic particle production, such as the dual unitarization model or reggeon calculus, are also tested by such $A$ dependences. The possibilities for $\frac{d N}{d y}$ of pions is shown in Fig. 6 .

In both the old and new scenarios, nucleon-nucleon interactions were assumed incoherent and fluctuations were therefore small. The Low-Nussinov model of hadron-hadron interactions, ${ }^{18}$ generalized to nucleus-nucleus interactions, ${ }^{13}$ provides a counter example to these scenarios. Nucleusnucleus collisions appear in close correspondence to hadron-hadron collisions within this model. In hadron-hadron collisions, there are large fluctuations event by event. Diffractive dissocation also may take place when a hadron projectile or target becomes excited with little or no particle production outside the fragmentation region. These phenomena have their counterpart in the Low-Nussinov model of nuclear collisions.

In this model, the nucleons in each nucleus change their color charge by a random walk in color space as they pass through one another. The average charge for such a walk is zero but $\left\langle Q^{2}\right\rangle \sim A$, the length of the 
walk. Since $\left\langle Q^{4}\right\rangle-\left\langle Q^{2}\right\rangle^{2} \sim A^{2}$, the fluctuations in $\left\langle Q^{2}\right\rangle$ are of order $\left\langle Q^{2}\right\rangle$. Since the amount of pair production in any event is a function of the charge squared of the nuclei, the fluctuations in multiplicity are large. These particles are pair produced by the two Lorentz contracted nuclei as they fly apart, and the computation of pair production is similar to that in QED for pair production in the presence of capacitor plates. $^{19}$ A string picture arises entirely as a consequence of the 1 dimensional geometry. ${ }^{13}$ Events which mock up diffractive dissociation appear when the two nuclei pass through one another and no local color charging takes place. The nuclei fragment, but no charge density produces a color electric field to drive pair production in the central region. This bizarre model of nucleus-nucleus collisions, however implausible, points out the danger of directly identifying high central region multiplicity with impact parameter zero collisions. These collisions are signaled by a violent nuclear break-up, and this may be correlated with central region multiplicity to check pictures of the collision dynamics. After - correlation is established, a central multiplicity trigger is justified. The attractive feature of this model is that large fluctuations may allow occasional events with large multiplicity and hence large energy density. If a proper impact parameter trigger is established, this modeI of the collision dynamics may be studied by the same techniques as in the standard scenario, since particles are produced in an inside-outside cascade and space, time and $\mathrm{p}^{\prime \prime}$ are correlated.

A primary reason for using heavy nuclei to produce new forms of matter is that the space-time volume of this matter should be sufficiently 
large for thermalization to occur. There are two types of thermalization: kinetic and chemical. Signals for chemical equilibration are discussed later in the discussion strange particle production. The issue of kinetic thermalization is whether a sufficiently large number of particle collisions take place so that the hadronic matter is in local thermal equilibrium. An interesting possible way of experimentally probing thermalization is by studying jet production. Bjorken has computed the $\frac{d E}{d x}$ energy loss for quarks in a quark-gluon plasma as $\frac{\mathrm{d} E}{\mathrm{dx}} \sim 10 \mathrm{GeV} / \mathrm{fm}$. A jet produced in such a plasma is quickly quenched. Unless the jet is produced near the surface of the plasma, it never makes it to a detector. The existence of single one sided jets would strongly suggest thermalization in nucleusnucleus collisions. A comparison of rates of jet production in nucleusnucleus collisions to that in hadron-hadron would probe the surface thickness of the plasma.

The size of the plasma space-time volume may be studied using a variety of hadronic and leptonic signals. The A dependences of production processes may isolate the space-time volume from which particles are emitted. The $P_{\perp}$ distributions may reflect the temperature and baryon number density at which the particles are dominantly produced. A temperature determination will have to be corrected for hydrodynamic expansion, and the effects of expansion may show up in the $p_{\perp}$ distributions. Since different hadron flavors have different cross sections and different mean free paths, flavor dependences may reflect different space time intervals for particle production. We shall soon discuss lepton probes in detail. 
Another kinematic region where the effects of thermalization might be seen is in the large $x$ portion of the fragmentation regions. ${ }^{6} \mathrm{~A}$ depletion at moderately large $x$ may reflect thermalization, but at extremely large $x$ there should be an excess since the plasma may emit hadrons well beyond the kinematic limit associated with the energy per nucleon. Such an effect must be corrected for fermi motion. An excess of $K^{\prime} s$ and $\Lambda^{\prime} s$ in the fragmentation region is later discussed as a signal for plasma formation.

Another probe of the space-time volume of a quark-gluon plasma might avise from studying quantum mechanical interferences of particles produced during the hadronization of the plasma. This subject has been extensively discussed by Willis. ${ }^{1}$ For nucleus-nucleus collisions, the density of produced particles is so high that this method may even be extended to multi-particle correlations. Although this method provides a potentially very exciting tool for analyzing the plasma, little theoretical work has been done to evaluate its utility for ultra-relativistic nuclear collisions.

The most striking confirmation of the existence of a quark-gluon plasma might be the existence of long-1ived, meta-stable droplets of quark-gluon matter. ${ }^{21-23}$ Such exotic forms of matter were first suggested in another context by Lee and Wick ${ }^{24}$. The possible existence of such meta-stable matter is made more plausible by the recent theoretical 25 and computational $^{26}$ results which strongly argue that the quark deconfinement transition is first order. The lifetime of these forms of quark-gluon matter might be anywhere from a few times the time it takes light to travel a fermi to macroscopic 1ifetimes. 
The most exciting (and implausible) situation would arise if quark-gluon matter was meta-stable for weak interaction lifetimes or longer. Such compressed hadronic matter would have a smaller cross section for fixed baryon number than would a corresponding nucleus. The binding energy of the matter would very probably be higher than that for a nucleus so that such a quark-gluon glob would form a penetrating hunk of hadronic matter. It would also probably have an anomolous charge to mass ratio, since the system might tend to neutralize itself by becoming strange.

These hypothetical quark-gluon globs might also be signalled by an explosive decay. This explosion might arise if the conversion to ordinary hadronic matter proceeded by detonation. The detonation of matter in a pipe is shown in $7 i g .7$. The detonation proceeds by a shock wave propagating supersonically through the super cooled media 2. Behind the shock front is heated ordinary hadronic matter. Immediately behind the shock front is a similarity rarefraction wave. If the detonation begins at the end of the pipe at $X=T=0$, the rarefraction wave carries momentum $P \sim X \sim T$. When the wave reaches the end of the pipe it ejects matter with a large momentum.

The formation of a spherical detonation wave might happen in a quark-gluon glob if a sufficiently large nucleation center of ordinary hadronic matter formed. The contents of the glob would convert to ordinary hadronic matter, and a significant portion of the glob mignt be ejected with large $p_{\perp}$. This matter might be baryon rich, since the 
glob itself in its ground state is baryon rich. The secondaries produced from the glob might have small $\mathrm{N}_{\pi} / \mathrm{N}_{\gamma}$, and might be composed of an anomolously large fraction of $\alpha$ particles. These secondaries would therefore have small mean free paths, many would have charge 2 and when they fragment, the tertiaries would have small $p_{\perp}$ and more normal mean free path.

This exotic and somewhat contrived scenario explains the main feutures of the Cëntauro and Chiron cosmic ray events. Although the $\mathrm{N}_{\pi} / \mathrm{N}_{\mathrm{B}}$ ratio predicted by the explosion of a glob may very well be too large to explain Centauros, and the Chirons may be fluctuations in conventional processes, these events do suggest some of the unconventional phenomena which might take place for ultra-relativistic heavy ion collisions. If Centauros are metastable quark-globs, a simple estimate based on the observed flux suggests they are produced once in $\sim 10^{4}$ nucleus-nucleus interactions at the top of the atmosphere.

A less exotic scenario for the mechanism in which a first order transition might show up is in the production of high $\mathrm{p}_{\perp}$ fireballs. This mechanism is suggested by Bjorken's work on $\overline{\mathrm{pp}}$ collisions and arises from considering detonation waves in a quark-gluon plasma. The discussion I present here was derived in collaboration with M. Gyulassy and correspond to established results for big bang cosmology. 30-31

In an ultra-relativistic nuclear collision a rapidly expanding hadronic matter distribution is produced. If this matter is a quark-gluon plasma and if the confinement-deconfinement phase transition is first order, 
the plasma might supercool. This supercooled plasma might convert to ordinary hadronic matter by forming detonation bubbles. This may happen if surface burning of the quark-gluon plasma does not have time to waste the plasma. 32

Detonation waves would have dramatic experimental consequences. They might be signalled by fluctuations in dN/ay over a unit or two of rapidity and large $p_{\perp}$. The average $p_{\perp}$ of the detonation waves increases as the nuclear radius $\sim \mathrm{A}^{1 / 3}$. The final state distribution of hadrons would be approximately azimuthally symmetric. A large fraction of the hadrons in ihe rapidity interval corresponding to the detonation bubble participate in the detonation. Some evidence for phenomena of this type are found in cosmic ray events. ${ }^{33}$ A useful way to look for the effects of such bubbles is with rapidity gap correlations, and azinuchal anglepseudorapidity distributions. 34

Even if the plasma does not convert to ordinary hadronic matter by detonation, large fluctuations in $\mathrm{dN} / \mathrm{dy}$ and correlations between azimuthal angle and pseudo-rapidity may be expected. Expanding matter distributions which undergo first order phase transitions typically produce large scale density fluctuations. The simplest example of this phenomena is the formation of rain. Even matter distributions which are not expanding often experience large scale density fluctuations, for example, boiling water. A theoretical study of the magnitude of density fluctuations under a variety of assumptions about nucleation and the strength of the phase transition might ciarify the status of such effects for nuclear collisions.

The study of fragments produced in the hydrodynamic expansion of matter produced in nuclear collisions may also have practical applications in nuclear physics and nuclear chemistry. In particular, the fragmentation 
region might have a reasonable fraction of strangeness rich hypernuclear fragments. Nuclei far from the island of stability might be produced. Perhaps even multi-meson bound states might be produced in the central region. The isolation and study of the spectroscopy of such hypothetical fragments may yield useful information about the nature of the nuclear force, especially for hyperons.

Studies of lepton and photon production may provide clean probes of the hadroric matter distribution produced in nuclear collisions. ${ }^{10,35-37}$ These leptons and photons are produced most copiously in the early stages of the collision when the matter is hottest, and penetrate the hadron matter distribution as a consequence of their long mean free paths. If leptons and photons from the plasma can be disentangled from those arising from incoherent hard quark scattering processes and conventional soft hadronic processes, ${ }^{3}$ the temperature and space-time volume of the plasma may be measured, and the quark-distributions in the plasma abstracted. The photons from $\pi^{\circ}$ decays provide a humongous background for plasma photons. Hard processes obscure plasma photons and leptons at high $\mathrm{P}_{\perp}$ and large inclusive di-lepton masses. Even at low $p_{\perp}$ backgrounds from conventional hadronic processes are potentially large. The magnitude of these effects relative to the plasma contributi $n$ is difficult to assess, due in part to the non-perturbative nature of the plasma contributions. Explicit perturbative computations are mildly encouraging that a sig al may arise if the correct kinematic range is studied. 
Assuming that a plasma contribution may be isolated, the $p_{\perp}$ distribution of photons and di-leptons indicates the primeval plasma temperature before expansion and cooling. The A dependence of the production rate gives infcrmation about the space-i:ime volume of the production process. A factor of A arises from the plasma volume. A factor of $t$ gives the time the plasma remains hot, and this may vary from $A^{1 / 3}$ to $\sim 1-2$ depending upon the model for the plasma expansion and whether the central region or fragmentation region is probed. Since the maximum plasma temperature grows as $\varepsilon \sim T^{4} \sim A^{1 / 3}$, the temperature of the plasma influences the A dependence of the total rate also. The study and analysis of these plasma photons and leptons will probably be involved. The possibility that a large increase in photon and lepton production occurs if the plasma forms is difficult to assess. Strange particle production might be enhanced if a quar:-gluon plasma is formed. ${ }^{39-41}$ In a hot plasma in chemical equilibrium, there are equal numbers of up, down, and strange quarks. Charm and higher mass quarks are suppressed at the temperatures relevant here. A difficulty with this scenario is that the plasma must live long enough that strange quarks come into equilibrium with up and down quarks, but this seems marginally to be true. ${ }^{40-41}$ In order to observe these strange quarks, they must survive expansion and hadronization. If the expansion is gentle enough so that not too many $\mathrm{K}^{\prime} \mathrm{s}$ are produced which subsequently decay into $K \pi$ systems and pollute a $K$ signal, ${ }^{14}$ copious kaons might be seen. Strange baryons might also arise, and the situation for final 
state pollution might be less severe since strange baryons preferentially decay by pion emission to strange baryons and eventually become $\Lambda^{\prime} s$.

An issue which has been ignored in this discussion is the possibility of a chiral symmetry restoration phase transition. ${ }^{42-43}$ There is now some weak evidence from lattice Monte-Garlo computations that this phase transition is another aspect of the confinement-deconfinement phase transition. ${ }^{26}$ The possibility that these transitions are distinct cannot be ruled out. ${ }^{42-43}$ Many of the probes discussed above for studying a plasma and phase changes carry over to the analysis of a chiral symmetry phase transition. A suggestion has been made that resonance widths might probe chiral symmetry restoration. ${ }^{43}$ since resonance widths are broadened by the presence of ordinary hadronic matter, this signal is a little hazy. Since the chiral symmetry phase transition is a relatively new subject, the future will probably bring a cornucopia of suggestions for probing this phase transition if it is in fact distinct from a confinementdeconfinement transition.

In ultra-relativistic heavy ion collisions, perhaps the unexpected is to be expected. This appears to be the case for the mysterious anomalons found at energies $\mathrm{E} / \mathrm{N} \sim 1-2 \mathrm{GeV} /$ nucleon $^{44-45}$. Evidence from these experiments suggests that incident nuclei such as iron may produce secondaries with a factor of 10 smaller mean free path than iron. There has been no entirely satisfactory explanation of this phenomenon. If anomalons are free quarks bound with a large number nucleons, 46,47 they would have fractional charge and this is not observed. ${ }^{48}$ A free gluon 
would probably be more difficult to produce than a free quark since a gluon has a higher color charge. If quark-gluon globs are produced, it would seem likely that the globs would have long mean free paths. The explanations of color polarized matter and toroidal nuclei seem difficult since the production cross section and lifetime of anomaloas are so large. Perhaps higher energy experiments will give some insight on this major loose end of Bevalac experiments. At the very least, anomalons provide an example of the variety of new phenomena and new theoretical Ideas which may be required to understand ultrarelativistic nuclear collisions. 


\section{References}

1. E. V. Shuryak, Phys. Rep. 61 (1980); Proceedings of Statistical Mechanics of Quarks and Hadrons, Bielefeld (1980); Proceedings of Workshop on Quark Matter Formation in Heavy Ion Collisions, Bielefeld (1982); Proceedings of Session on Heavy Ions, Moriond Conf. (1982) in Phys. Rept. 88, 379 (1982).

2. Proposal for a $15 \mathrm{~A}-\mathrm{GeV}$ Heavy Ion Facility at Brookhaven, BNL-32250.

3. L. McLerran, Proceedings of High Energy Heavy Ion Study, Berkeley (1981).

4. A. Mueller, Proceedings of 1981 Isabelle Summer Study.

5. J. Bjorken, Fermilab-Pub-82/44-THY (1982).

6. J. Cleymans, M. Dechantstreiter, and F. Halzen, Wisconsin preprint Mad-Th-50 (1982).

7. R. Anishetty, P. Koehler, and L. McLerran, Phys. Rev. D22, 2793 (1980).

8. K. Kajantie and L. McLerran, Phys. Lett. 119B, 203 (1982); Helsinki preprint HU-TFT-82-30 (1982).

9. L. D. Landau, Izv. Akad. Nauk. Sec. Fiz. 17, 51 (1953); "Collected Papers of L. D. Landau", D. Ter Haar, Editor, Pergamon Press, Oxford (1965).

10. E. V. Shuryak in reference 1 .

11. W. Busza and A. S. Goldhaber, MIT Preprint ITP-SB-82-22 (1982).

12. J. Kapusta, U. Minnesota preprint, Print-82-0892 (1982).

13. H. Ehtamo, J. Lindfois and L. McLerran, U. Helsinki preprint.

14. J. Bjorken in "Current Induced Jeactions", J. G. Korner, G. Kramer, D. Schitdhsecht, Epringer Verlag, Berlin (1976); Proceedings of 2ud Int. Conf. on Physics in Collisions: High Energy echepl pp Interactions Stockholm (1982). 
15. K. Kajantie and R. Raitio, Helsinki preprint, HU-TFT-82-52 (1982).

16. M. Gyulassy, LBL report, LBL-15175 (1982).

17. JACEE collaboration, in Proceedings of Workshop on Very High Energy Cosmic Ray Interaction, U. Penn., April, 1982.

18. F. E. Low, Phys. Rev. D12, 163 (1975); S. Nussinov, Phys. Rev. Lett. 34, $1286(1976)$.

19. J. Kogut and L. Susskind, Phys. Rev. D9, 3501 (1974).

20. J. D. Bjorken, Fermilab preprint FNAL-Pub-82/59-THY (1982).

21. S. A. Chin and A. Kerman, Phys. Rev. Lett. 43, 1292 (1979).

22. J. D. Bjorken and L. D. McLerran, Phys. ReV. D20, 2353 (1979).

23. A. K. Mann and H. Primakoff, Phys. Rev. D22, 1115 (1.980).

24. T. D. Lee and G. C. Wick, Phys. Rev. D9, 2291 (1974).

25. L. G. Yaffe and B. Svetitsky, Phys. Rev. D26, 963 (1982).

26. J. Kogut et al., Illinois preprint, III-TH-82-39.

27. J. Chinellato et al., "Centauros" Report of Science and Engineering, Laboratory of Waseda University.

28. J. Chinellato et al., "Chirons" Report of Science and Engineering, Laboratory of Waseda University.

29. J. Bjorken, private communication and Ref. 20 .

30. P. J. Steinhardt, Phys. Rev. D25, 2074 (1982).

31. M. Gyulassy and L. McLerran, in preparation.

32. M. Danos and J. Rafelski, Frankfurt preprint UFTP-94 (1982).

33. J. Iwai et a1., Nuovo Cimento A69, 295 (1982).

34. J. Wilkes, private communication.

35. E. Feinberg, Nuovo Cimento 
36. G. Domokos and J. I. Goldman, Phys. Rev. D23, 203 (1981).

37. K. Kajantie and H. Miettinen, Z. Phys. C9, 341 (1981).

38. F. Halzen and H. C. Liu, Phys. Rev. D25, 1842 (1982).

39. J. Rafelski, Phys. Rept. 88, 331 (1982).

40. J. Rafelski and B. Muller, Phys. Rev. Lett. 48 (1982).

41. T. S. Bero, B. Lukacs, J. Zimanyi, and H. W. Barz, Nucl. Phys. A386, 617 (1982).

42. E. V. Shuryak, Phys. Lett. 107B, 103 (1981).

43. R. Pisarski, Phys. Lett. 110B, 155 (1982).

44. B. Judek, Proceedings 14th International Cosmic Ray Conference, Vo1. 7, Muenchen (1975).

45. E. M. Friedlander et al., Phys. Rev. Lett. 45, 1084 (1980).

46. G. Shan and R. Slansky, Irvine preprint UCI-82-46 (1982). 


\section{Figure Captions}

Fig. 1 Two Lorentz contracted nuclei approach one another in the center-of-mass frame.

Fig. 2 The two nuclei stick together.

Fig. 3 A hypothetical baryon charge rapidity distribution for the old scenario for nucleus-nucleus collisions.

Fig. 4 The two nuclei pass through one another and matter begins to form.

Fig. $5 \quad \mathrm{dN} / \mathrm{dy}$ for baryons minus antibaryons in the new scenario.

Fig. 6 The possible distributions of pions.

Fig. 7 Detonation in a pipe. 

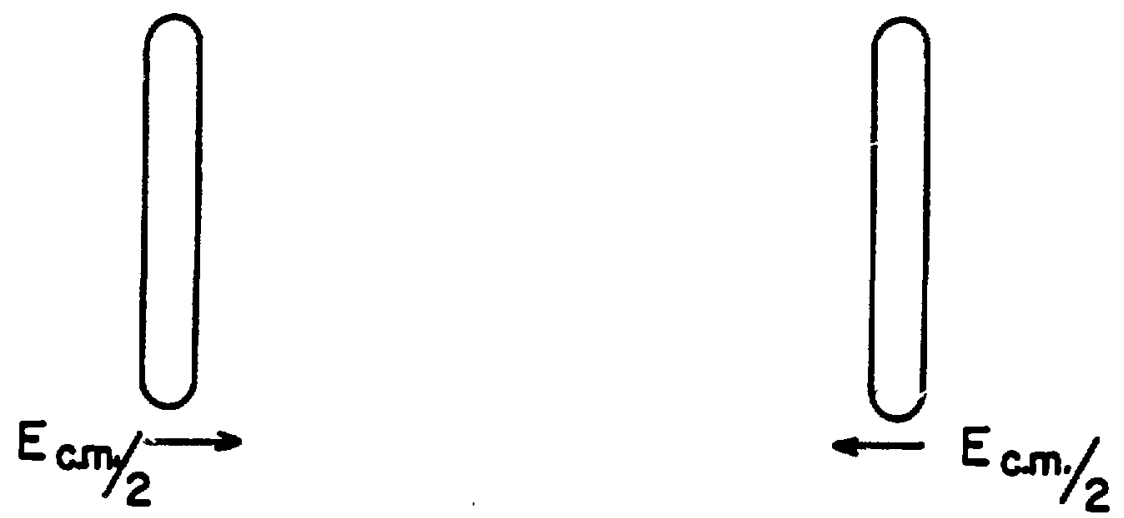

Figure 1

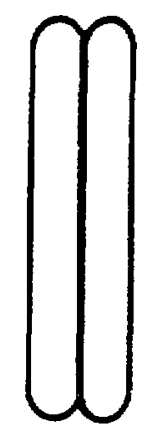

Figure 2 


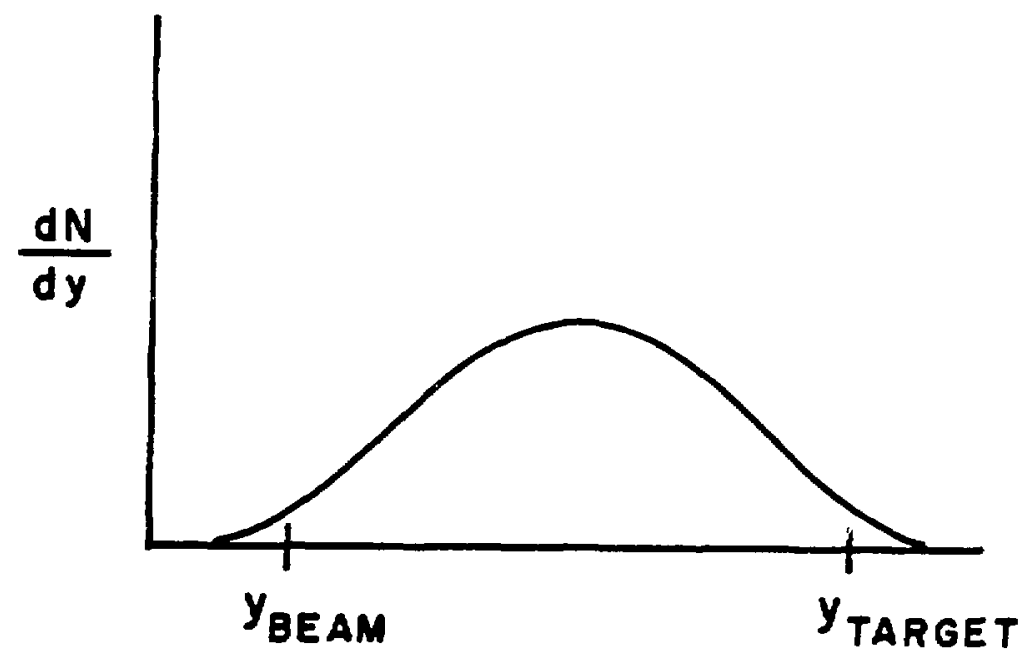

Figure 3

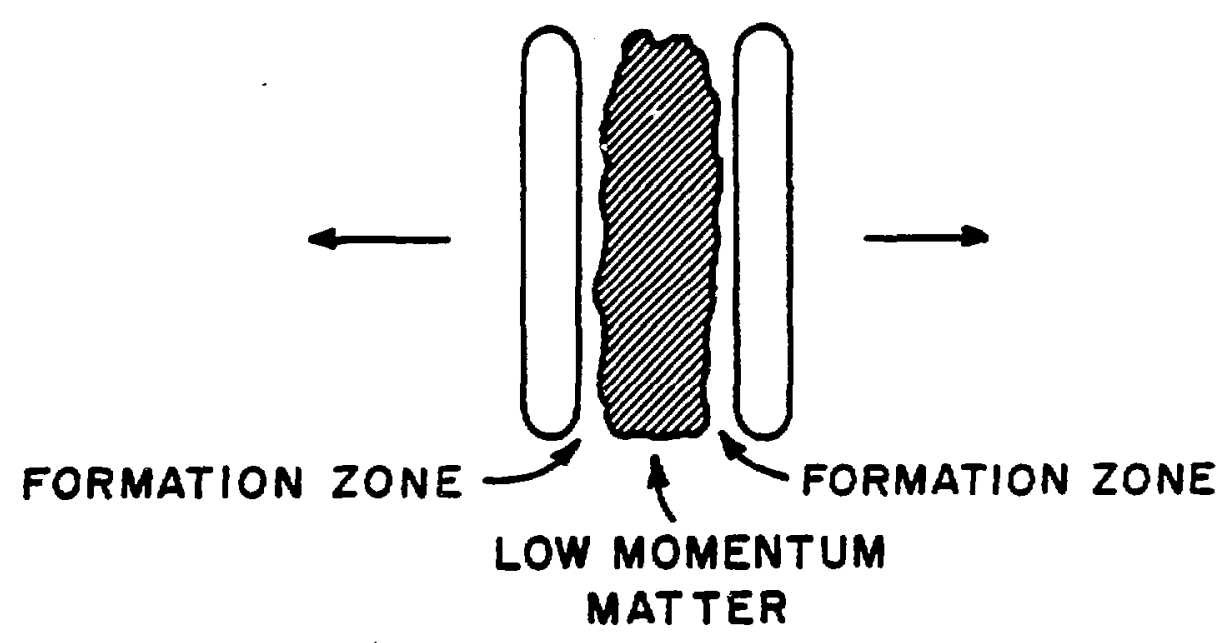

Figure 4 


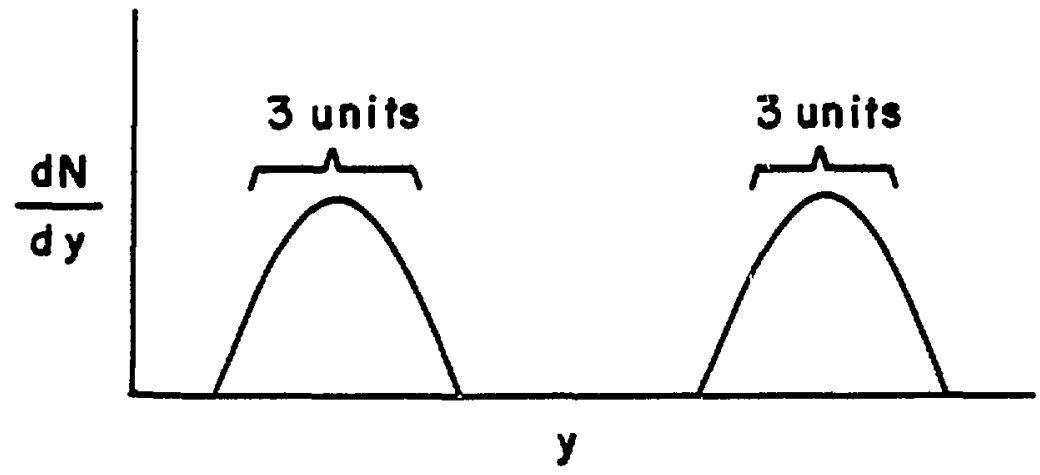

Figure 5

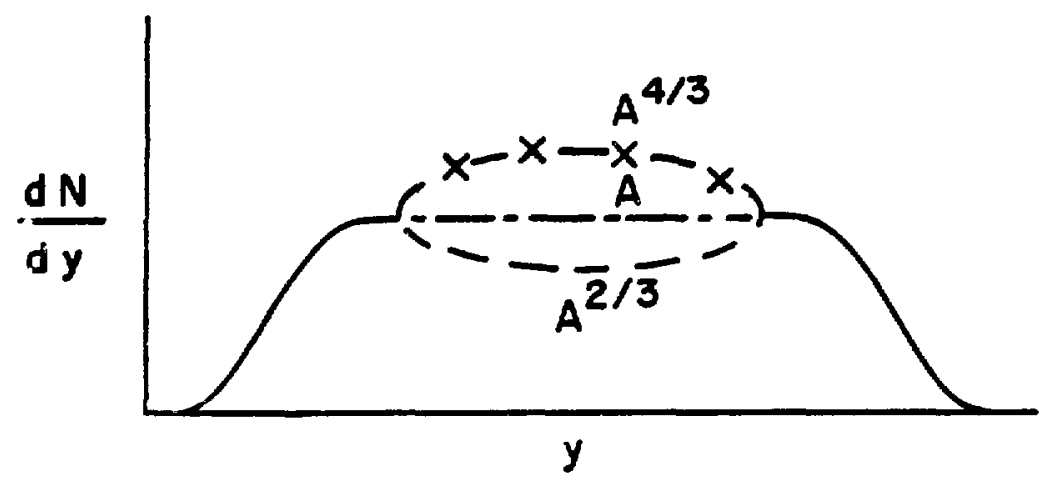

Figure 6 


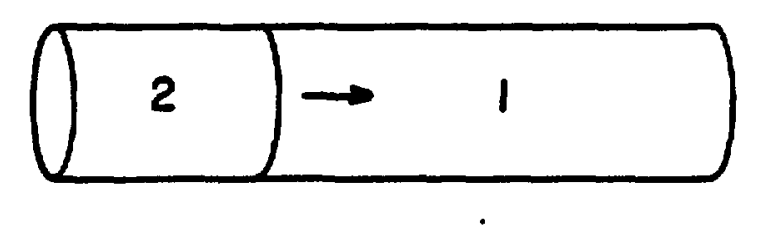

Figure 7 
NOTICE

This report was prepared as an account of work sponsored by the United States Government. Neither the United States Government nor any agency thereof, nor any of their employees, makes any warranty, express or implied, or assumes any legal liability or responsibility for the accuracy, completeness, or usefulness of any information, apparatus, product or process disclosed, or represents that its use would not infringe privately owned rights. Reference herein to any specific commercial product, process, or service by trade name, trademark, manufacturer, or otherwise, does not necessarily constitute or imply its endorsement, recommendation, or favoring by the United States Government or any agency thereof. The views and opinions of authors expressed herein do not necessarily state or reflect those of the United States Covernment or any agency thereof. 